\title{
Social games in a social network
}

\author{
Guillermo Abramson ${ }^{1,2} *$ and Marcelo Kuperman ${ }^{1} \dagger$ \\ ${ }^{1}$ Centro Atómico Bariloche and Instituto Balseiro, 8400 S. C. de Bariloche, Argentina \\ ${ }^{2}$ Consejo Nacional de Investigaciones Científicas y Técnicas, Argentina
}

We study an evolutionary version of the Prisoner's Dilemma game, played by agents placed in a small-world network. Agents are able to change their strategy, imitating that of the most successful neighbor. We observe that different topologies, ranging from regular lattices to random graphs, produce a variety of emergent behaviors. This is a contribution towards the study of social phenomena and transitions governed by the topology of the community.

PACS numbers: 87.23.Ge, 02.50.Le, 87.23.Kg

The search for models that account for the complex behavior of biological, social and economic systems has been the motivation of much interdisciplinary work in the last decade [1]. In particular, the emergence of altruistic or cooperative behavior is a favorite problem of game theoretical approaches [2]. In this context, the Prisoner's Dilemma game [3] has been widely studied in different versions, as a standard model for the confrontation between cooperative and selfish behaviors, the later manifested by a defecting attitude, aspiring to obtain the greatest benefit from the interaction with another individual. It is usually implemented in zero dimensional systems, where every player can interact with any other. It has also been studied on a regular lattice, where a player can interact with its nearest neighbors in an array 槒. In a regular lattice the concept of a $k$-neighborhood is straightforward. It is composed of the $k$ nearest individuals to a given one. However, social situations are rarely well described by such extreme networks. The topology of social communities is much better described by what has been called small-world networks [5,6]. In the version of small worlds that we use in this work, the "regular" $k$-neighborhood of an individual is modified by breaking a fraction of its $k$ original links. An equal amount of new links are created, adding to the neighborhood a set of individuals randomly selected from the whole system.

We have studied a simple model of an evolutionary version of the Prisoner's Dilemma game played in smallworld networks. The Prisoner's Dilemma was chosen as a paradigm of a system capable to display both cooperative and competitive behaviors [7]. The evolutionary dynamics is implemented by an imitation behavior. It is im-

*E-mail address: abramson@cab.cnea.gov.ar

${ }^{\dagger}$ E-mail address: kuperman@cab.cnea.gov.ar portant to notice that, contrary to the iterated version of the game, our players do not remember past encounters. The emergence of cooperation is only due to the spatial organization of the players. This is the main rationale behind the study of the influence of the topological aspects of the community. We have explored different topologies, ranging from a regular lattice to random graphs, going through the small worlds. We have found surprising collective behaviors corresponding to the small-world systems, put on evidence by the enhancement of defection in situations where cooperation is the norm.

We set up a system of $N$ players arranged at the vertices of a network (as described below). Each player is a pure strategist, adopting either a cooperative or a defecting strategy. Each vertex is connected, on average, to other $2 K$ vertices, that we call its neighbors. The edges connecting two vertices enable the interaction between players. A round of play consists in the confrontation of every player with all its neighbors. Each one of these produces a profit for the player, computed with the payoff matrix:

$$
\begin{array}{c|c|c} 
& \mathbf{C} & \mathbf{D} \\
\hline \mathbf{C} & r & s \\
\hline \mathbf{D} & t & p
\end{array}
$$

Each element in the payoff matrix represents the payoff of a player performing the strategy at left, when confronting a player that performs the strategy above. A defector $\mathbf{D}$ obtains $t$ - the temptation to defect-when its opponent is a cooperator $(\mathbf{C})$, who gets $s$ - the sucker's payoff. Each of two cooperators obtains a reward $r$, while each of two defectors is punished with $p$. The Prisoner's Dilemma is played with $t>r>p>s$ and $2 r>t+s$. In this work we use a simplified version of the game, assuming that $s=p=0, r=1$. All the interesting features of the game are preserved [- 4 , and we are left with a single parameter to play with.

Let's represent the players with two-component vectors $x$, taking the values $(1,0)$ for $\mathbf{C}$-strategists and $(0,1)$ for D-strategists. The payoff matrix is:

$$
\mathbf{A}=\left(\begin{array}{ll}
1 & 0 \\
t & 0
\end{array}\right)
$$

As a result of the confrontation with its neighbors in a single round, at time $\tau$, each player collects a payoff

$$
P_{i}(\tau)=\sum_{j \in \Omega_{i}} x_{i} \mathbf{A} x_{j}^{T}
$$


where $\Omega_{i}$ is the set of neighbors of element $i . P_{i}$ is the profit earned by a player in a time step, and it is not accumulated from round to round.

After this, the players are allowed to inspect the profit collected by its neighbors in that round, adopting the strategy of the wealthiest among them for the next round of play. If there is a draw between more than one neighbor, one of them is chosen at random to be imitated. If the element under consideration is itself one of the winners of the round, it keeps its own strategy. That is, explicitly writing the time dependence of the strategies:

$x_{i}(\tau+1)=\left\{\begin{array}{l}x_{i}(\tau) \text { if } P_{i}(\tau) \geq \max \left(P \in \Omega_{i}\right) \\ x_{j}(\tau) \text { if } j \in \Omega_{i} \text { and } P_{j}(\tau)=\max \left(P \in \Omega_{i}\right) .\end{array}\right.$

We have found that a small amount of noise is essential to prevent the system from falling in a frozen state. After a round of play, we chose one element at random and flip its strategy. This is enough to keep the system out of equilibrium and allow transitions between different states.

As a playground for our system, we have used a family of small-world networks that depend on a parameter $\epsilon$ [6]. We start from a regular, one-dimensional, periodic lattice of coordination number $2 K$. We then run sequentially through each of the sites, rewiring $K$ of its links with probability $\epsilon$. Running from 0 to 1 , this parameter changes the wiring properties of the network, ranging from a completely ordered lattice at $\epsilon=0$, to a random network at $\epsilon=1$. Intermediate values of $\epsilon$ produce a continuous spectrum of small-world networks. Double connections between sites, as well as the connection of a site with itself, are avoided in the construction of the network. Since we neither destroy nor create links, the resulting network has an average coordination number $2 K$, equal to the initial one. This method, however, can produce disconnected graphs, that we have avoided in our analysis. Note that $\epsilon$ is related to the fraction of modified regular links.

Two magnitudes characterize the topological properties of the small-world networks generated by the indicated procedure. One of them, $L(\epsilon)$, measures the typical separation between any pair of elements in the network. The other, $C(\epsilon)$, measures the clustering of an element's neighborhood [6]. Ordered lattices are highly clustered, and have large $L$. Random graphs have short characteristic length and small clusterization. In between, small worlds can be characterized by a high clusterization (like lattices) and short path lengths (like random networks).

The opposing tendencies of cooperation and defection perform differently for different payoff tables and different topologies, through the values of $t$ and $\epsilon$. Disregarding $\epsilon$, one may qualitatively expect that, for sufficiently high values of $t$ it would pay to defect while, for low values of $t$, it would be worth to cooperate. In either of these two extremes, the system would collapse to a state formed only by defectors (in the first case) or only by cooperators (in the second case). For intermediate values of $t$ the system would settle into a mixed state consisting in cooperators and defectors. Cooperators would thrive through the formation of clusters, that can resist the invasion by defectors. The dependency on the topology of the network appears on top of these three regimes. From the structure of the payoff matrix one may conjecture that the high values of $t$ referred to above will be around $t=2$ (where a defector earns twice as much as a pair of cooperators). Correspondingly, the low values of $t$ will be around $t=1$ (where a single cooperator earns more than a defector).

In the following we show the results of simulations performed in systems with 1000 elements. The initial strategies are assigned at random with equal probability. Then several hundred rounds are played to allow for an asymptotic regime to be achieved. All the results shown are averages over realizations where both the networks and the initial conditions are randomly chosen, excluding all disconnected graphs from our analysis.

Fig. 1 Abramson \& Kuperman Social games in a social network

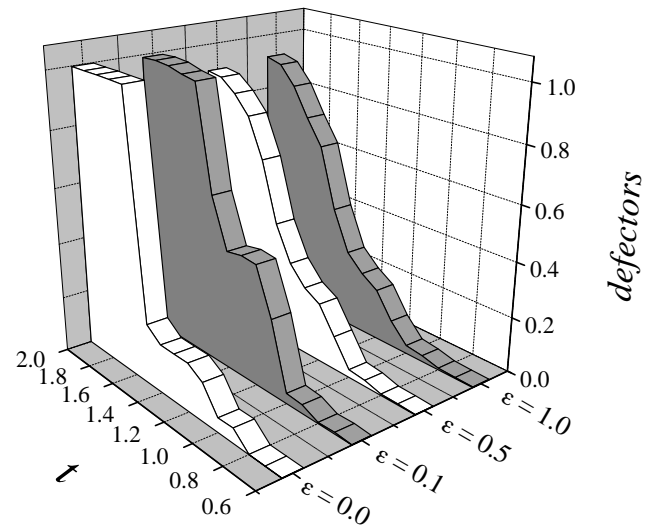

FIG. 1. Fraction of defectors as a function of the temptation to defect $t$, for different values of the rewiring probability $\epsilon$ (as shown in the legend). The results correspond to 10 independent realizations of 1000 elements, run for 500 rounds after a transient of 200 rounds. Note that the range of $t$ extends to values lower than 1 , where the game is not a proper Prisoner's Dilemma.

The number of cooperators and defectors are fluctuating variables, with bell shaped distributions. In figure 1 we show the average fraction of defectors in systems with $K=2$, that is, systems with an average coordination number of four. Four curves are shown as a function of the parameter $t$. Each curve corresponds to a network characterized by the parameter $\epsilon$ shown in the legend. All the curves show a growth in the fraction of defectors for growing values of $t$, as expected. We can see however, that the small world corresponding to $\epsilon=0.1$ displays an enhanced number of defectors at values of $t$ around 1.2. For systems with a fixed $K$ and a fixed $t$, this means 
that the existence of a small world topology with $\epsilon \sim 0.1$ represents that nearly $40 \%$ of the population adopts the defecting strategy, against the $20 \%$ of more regular or more random networks. (Note that, in fig. 1, we have included values of $t$ lower than 1 , where the game is not a proper Prisoner's Dilemma, since the reward for cooperation is greater that the temptation to defect. We have done so because the state of the system at $t=1$, for all values of $\epsilon$, still contains a small fraction of defectors. We wanted to stress that for low enough values of $t$ the state is complete cooperation.)

Fig. 2 Abramson \& Kuperman Social games in a social network

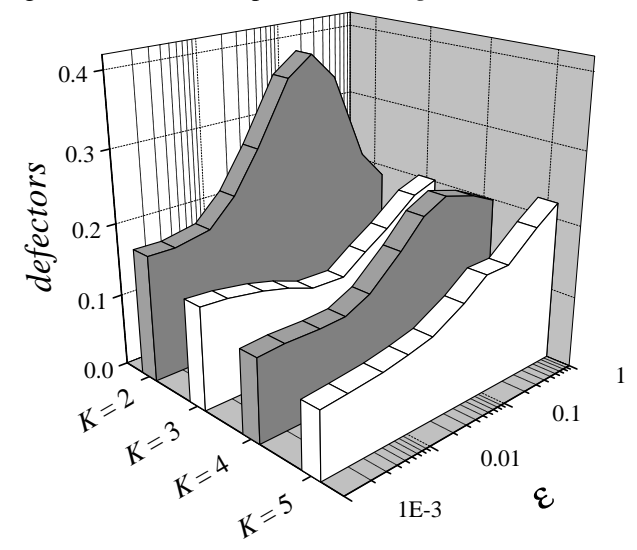

FIG. 2. Fraction of defectors as a function of the rewiring probability $\epsilon$, for different values of $K$ (as shown in the legend). The results are derived from 10 independent realizations of 1000 elements, run for 1000 rounds after a transient of 1000 rounds. Games played with $t=1.2$

In figure 2 we show a plot of the same system, but with the fraction of defectors as a function of $\epsilon$, to emphasize the changes in behavior as the structure of the network varies. The four curves correspond to different coordination numbers. The game corresponds to the value $t=1.2$ in the payoff matrix, so that the curve with $K=2$ is a slice of figure 1 cut at $t=1.2$. Note that only this curve has a clear high peak of defectors centered near $\epsilon=0.1$. Systems with $K=3$ have a downward peak instead, in the region of small worlds, indicating a slight enhancement of the cooperative strategy. For $K=4$ we can see again a small peak of defectors. Systems with $K=5$ and greater (not shown) display a monotonous behavior in $\epsilon$.

Some conjecture on the origin of these features may be appropriate here. We think that the competition between the stability of clusters of cooperators and their exploitation by neighbor defectors at the borders contributes to the features observed here. When $K=2$, the cooperators survive in small compact groups. As $\epsilon$ grows, these groups can be formed by elements widely dispersed in the system, where they will have more defector neighbors to compete with. In this way, there will be less configurations to support them and, consequently, more defectors in the system. For $\epsilon$ even greater, and more long range links, cooperators may start to reconnect and survive the competition with the defectors. When $K>2$, the cooperators can only survive in larger groups, because defecting neighbors at the border of a group can penetrate deeper. When $\epsilon$ grows, cooperators belonging to faraway groups may become connected to form large clusters able to survive. The fact that $K=3$, at $t=1.2$ shows a slight decrease in the fraction of defectors at intermediate values of $\epsilon$ remains, however, unexplained in this picture. At other values of $t$, we observed that the system with $K=3$ performs like that with $K=2$, namely with an enhancement of defector at intermediate values of $\epsilon$.

Fig. 3 Abramson \& Kuperman Social games in a social network

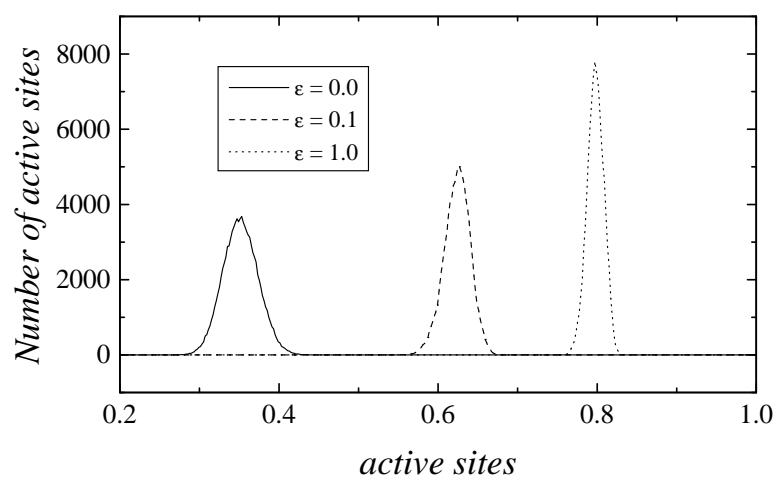

FIG. 3. Distribution of the fraction of "unsatisfied" or active elements, for different values of the rewiring probability $\epsilon$ (as shown in the legend). The results correspond to 100 independent realizations of 1000 elements, run for 1000 rounds after a transient of 1000 rounds, with $t=1.2$.

Also, we have observed that, for every system, there is a fluctuating number of "unsatisfied" elements that change their strategy. There is a Gaussian distribution of these unsatisfied elements, whose mean increases with $\epsilon$, as shown in figure 3. This behavior is observed for all values of $K$ and of $t$, namely that regular lattices contain a smaller number of unsatisfied elements than random networks, with small worlds in between.

Most of what is analytically known about small worlds refers to the distribution of shortest paths between pairs of elements (see for example [8, 10]). It is known that regular lattices stand apart from even infinitesimally rewired small worlds, that behave like random networks. The existence of a phenomenon like the enhancement of defectors density at a finite value of $\epsilon$, as shown in this work, points to the existence of an interesting phenomenology in small worlds. The broad spectrum of behaviors of a given system as a function of the topological features of the network is the main aspect that we want to emphasize. This suggests the possibility of modelling a certain system featuring well known interactions and analyzing the influence of the particular organization the commu- 
nity. Moreover, the possibility of a self organizing network with changing links opens the possibility of modelling more realistically social and economical situations [11]. At this point we can state that the self organization of the network can lead to a nontrivial behavior of the whole system. Another interesting example of this statement would be a simple SIR model for the propagation of an epidemic. This is the subject of work under way [12].

The authors thank Damián H. Zanette for interesting discussions.

[1] H. Haken, Synergetics, an introduction (Springer, Berlin, 1978). T. Poston and I. Stewart, Catastrophe theory and its applications (Pitman, London, 1978).

[2] J. Maynard Smith, Evolution and the Theory of Games (Cambridge University Press, Cambridge, UK, 1982).

[3] R. Axelrod and W. D. Hamilton, Science 211, 1390-1396 (1981).

[4] M. A. Nowak and R. M. May, Nature 359, 826-829 (1992). M. A. Nowak and R. M. May, Int. Jour. Bif. Chaos 3, 35 (1993).

[5] S. Milgram, Psychol. Today 2, 60-67 (1967).

[6] D. J. Watts and S. H. Strogatz, Nature 393, 440-442 (1998).

[7] R. Axelrod, The Complexity of Cooperation (Princeton University Press, Princeton, NJ, 1997).

[8] M. E. J. Newman and D. J. Watts, Phys. Lett. A 263, 341 (1999).

[9] M. E. J. Newman, C. Moore and D. J. Watts, Phys. Rev. Lett. 84, 3201 (2000).

[10] C. F. Moukarzel, Phys. Rev. E 60, R6263 (1999).

[11] M. G. Zimmermann et al., "Emergence of cooperation in an adaptive network with local interactions" (to be published).

[12] M. Kuperman and G. Abramson, "Small world effect in an epidemiological model," available as hlin.AO/0010012 (2000). 